\title{
Comparison of thyroid profile in premenopausal and postmenopausal women: A case control study
}

\author{
G.S Chandrashekhar ${ }^{1}$ \\ ${ }^{1}$ Dr. G.S Chandrashekhar, Senior Physician \& Cardiodiabetologist, Medical Director, Adarsha Super Speciality Hospital, \\ Udupi, Karnataka, India.
}

Corresponding Author: Dr. G.S Chandrashekhar, Senior Physician \& Cardiodiabetologist, Medical Director, Adarsha Multi Speciality Hospital, Udupi, Karnataka. India. E-mail: drgsc0606@gmail.com

\begin{abstract}
Introduction : After menopause, diminishedgonadal hormones are found to influence thyroid status. There are a few published literature in this area, to the best of our knowledge. Aim of the study was to compare thyroid profile of postmenopausal women with those in reproductive age group as well as to find the correlation between age and thyroid profile. compare thyroid profiles of young men with those in geriatric age group iii. find if anygender differences in thyroid profiles. Methods: In a case control study, thyroid profiles of 76 post menopausal women and 131 women in reproductive age group were compared. Thyroid profile of 78 young men was compared with that of 24 men in geriatric age group. Gender differences in thyroid profile were studied by comparing thyroid profiles of 207 women and 102 men. Statistical analysis was carried out using MannWhitney U test and Linear regression analysis. Results: A significantly higher TSH levels $(\mathrm{P}<0.05)$ and decreased T3 $(\mathrm{P}<0.0001)$ were observed in postmenopausal women compared to the controls. There was no statistically significant difference in thyroid profiles among men in geriatric age group and young men. There was a significant elevation of TSH in women compared to men $(\mathrm{P}<0.05)$. A negative correlation between age and T3 levels was observed in women. Conclusion: Subclinical hypothyroidism was observed in post menopausal women in our setting. A higher tendency of women having subclinical hypothyroidism compared to men was also observed. This suggestsa necessity to assess functional status of thyroid gland periodically in the post menopausal period.
\end{abstract}

Key words: Thyroid profile, Menopause, Gender difference

\section{Introduction}

Menopauseis a condition where there is cessation of menstruation under the influence of reproductive hormones. The menopause is characterized by wide variability in both FSH and estradiol in the blood. In menopause, the ovaries producelessestrogen and these changes in estrogen exposure have important effects on most tissues in the body.

Endocrine glands exhibit changes as the age advances. Age related fall in circulating T4, reduction in $\mathrm{TSH}$ bioavailability, reduced responsiveness of thyroid gland to TSH can result in increased TSH secretion in the absence of overt thyroid disease. Subclinical hypothyroidism with normal free T4 and raised TSH is found to be common in elderly [1,2]. Gender specific alterations in TSH and FT 4 has also been reported in which elderly women had elevated TSH, without any

Manuscript received: $6^{\text {th }}$ October 2018

Reviewed: $16^{\text {th }}$ October 2018

Author Corrected: $24^{\text {th }}$ October 2018

Accepted for Publication: $30^{\text {th }}$ October 2018 alterations in FT4 [3]. These hormones influence other systems in the body as well. Thyroid gland is one among them. Thyroid hormone regulates body metabolism, including reproductive functions. Disorders of thyroid gland are common in women. We could finda few American studies in which higher TSH levels were reported in post menopausal women [4,5]. One of the previous studies suggested an elevation of TSH in postmenopausal women [6]. There is a scarcity of literature in Indian settings which focus on thyroid status in post menopausal women.

\section{Objectives}

Aims of this study were to compare thyroid profile of postmenopausal women with those in reproductive age group as well as to find the correlation between age and thyroid profile. To compare thyroid profiles of young men with those in geriatric age group\& to find if anygender differences in thyroid profiles. 


\section{Methodology}

Study design- The case control study was conducted in Adarsha Hospital, Udupi. Ethics committee approval was sought before starting the study.

Inclusion criteria: Thyroid profiles of 207 women were studied, out of which 131 were in reproductive age group, 76 were postmenopausal. Thyroid profiles of 102 men were assessed, out of which 24 were in geriatric age group and 78 were young men.

Mean ages of premenopausal and post menopausal women were $34.91 \pm 0.91$ years and $62.63 \pm 1.15$ years respectively.

Mean ages of young and geriatric men were $42.06 \pm 1.57$ years and $71 \pm 1.49$ years respectively. Overall mean age of women and men were $45.09 \pm 1.17$ years and $48.87 \pm 1.75$ years respectively.
Exclusion criteria for cases: diabetics, hypertensives, known thyroid disorders

Sample collection and analysis- Five $\mathrm{ml}$ of venous blood sample was collectedin plain tubes by puncturing antecubital vein with aseptic precautions. Samples were centrifuged at $3000 \mathrm{rpm}$ for 15 minutes. Serum was analyzed for thyroid hormones and TSH with commercially available kits in hormone analyzer Mini Vidas, which works on the principle of Immunofluorescence.

Statistical analysis- Statistical analysis was carried out using the software SPSS 16. Mann Whitney U test was used to compare thyroid profiles. Correlation of thyroid profile with age was computed by linear regression analysis.

\section{Results}

A significantly higher TSH levels $(\mathrm{P}<0.05)$ and decreased $\mathrm{T} 3(\mathrm{P}<0.001)$ were observed in postmenopausal women compared to the controls (fig 1). There was no statistically significant difference in thyroid profiles among men in geriatric age group and young men $(\mathrm{P}>0.05)$ (fig 2$)$.

However TSH was insignificantly high in geriatric group. There was no significant gender difference in thyroid profile $(\mathrm{P}>0.05)$ except for serum TSH levels which was significantly high in women $(\mathrm{P}<0.05)($ fig 3$)$.

There was a significant negative correlation between age and T3 levels in women (correlation coefficient $(r)=-0.2018 . \mathrm{r}^{2}$ $=0.04074 ; \mathrm{P}=0.0035)$.

There was no significant deviation of runs from the linearity (Fig 4). There was no significant correlation between age and T4, TSH $(\mathrm{P}>0.05)$

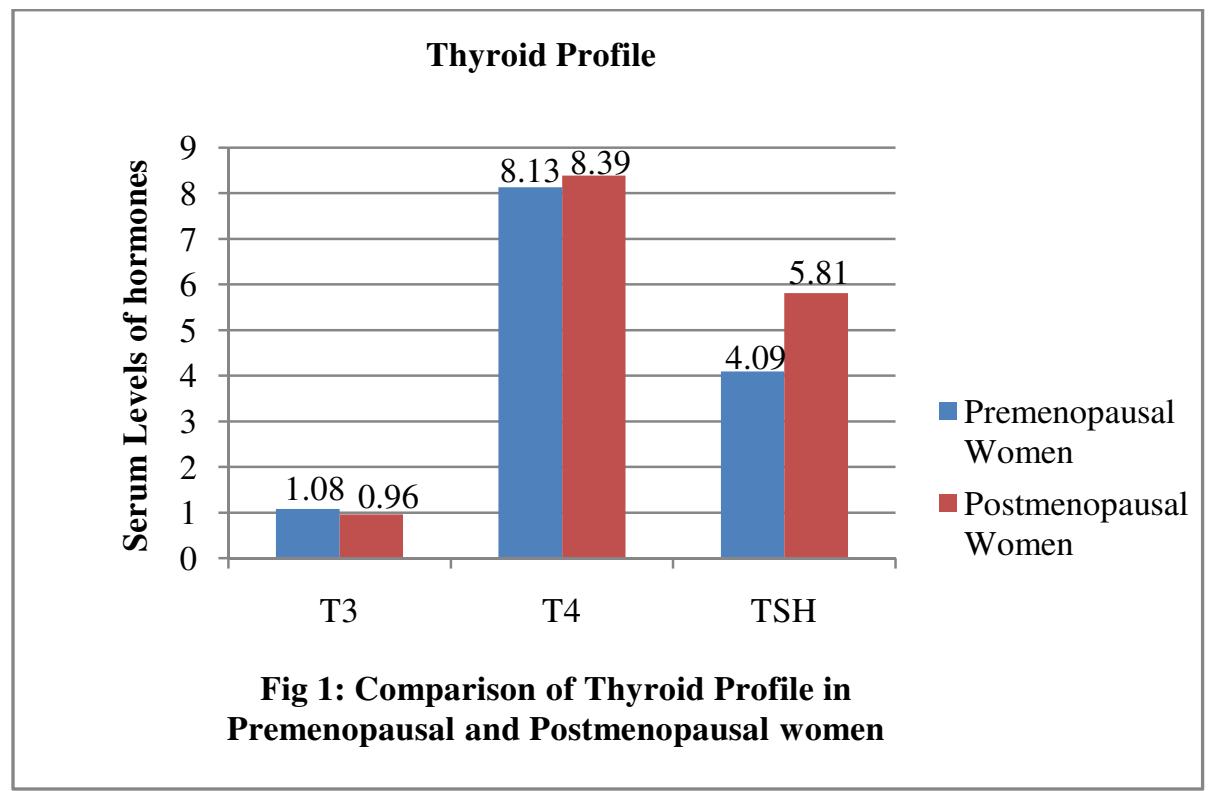


Original Research Article
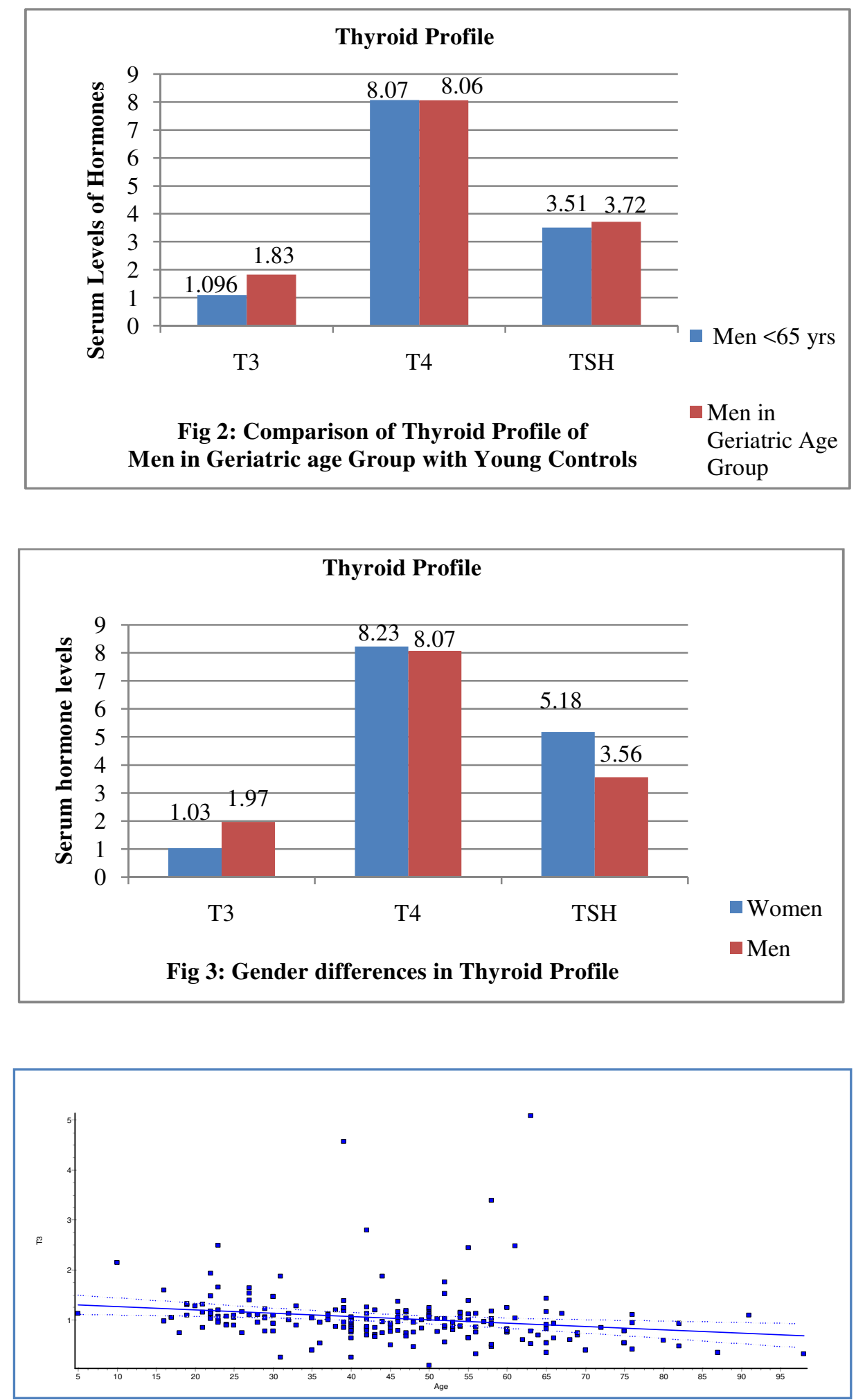

Fig 4: Linear regression correlation analysis of Age and T3.

\section{Discussion}

A significant elevation in TSH and decline in T3 levels were observed in post menopausal women (fig 1). Study by Adiga et alfound a significant elevation in TSH levels $(\mathrm{P}<0.05)$ as well as no significant decline in thyroid hormones in post menopausal women compared to those in reproductive age group [6]. A positive
Correlation has also been found between TSH and age. Similar results were observed by Rojas et al, Pearce et al and Hollowell et al [4,5,7]. Altered thyroid functions can be explained as an age-related fall in thyroid hormones or reduced responsiveness of thyroid to TSH in the absence of overt thyroid disease. It also may be 


\section{Original Research Article}

an occult thyroid disease or just an age-related alteration in TSH set point $[8,9]$. However contradictory reports are available, which suggest a lower TSH levels in post menopausal women $[10,11]$.

Thewomen healthrelies in a delicate balance of hormones, such as estrogen, progesterone, and testosterone. The thyroid hormones influence the functioning of all major organs along with reproductive system development and overall body metabolism. In women, diseases of the thyroid gland are among the most prevalent disorders world wide, second only to diabetes [12]. With time, overt hypothyroidism can develop in menopausal age, the symptoms of which can be similar to postmenopausal complaints and are clinically difficult to differentiate. There can also be an absence of clinical symptoms.

Elevated TSH in elderly, especially in women can be physiological or pathological. History of nutritional status, associated illness and follow up with TSH measurement helps to differentiate.

The American Thyroid Association (ATA), the Endocrine Society and the American Association of Clinical Endocrinologists (AACE) had recommended aggressive case finding in elderly women. Thyroid stimulating hormone (TSH) is the preferred test to assess thyroid function as stated by National Academy of Clinical Biochemistry [13]. It has been reported by the 2012 clinical practice guidelines co-sponsored by the AACE and ATA that the serum TSH is the single best screening test for primary vast majority of out patient clinical situations [14]. It is evident from the various study results that periodic screening of post menopausal women for thyroid dysfunction is very much needed.

We could not demonstrate any significant alterations in thyroid status of elderly compared to young adults due to the small sample size of geriatric population. However TSH has been reported to be increased in elderly. The causes may be anti-thyroid antibodies, nutritional deficiency of iodine, hidden thyroid autonomy etc. Sleep disturbances and altered sleep patterns with increasing age may lead to increase in TSH levels [15]. Aging is associated with changes in pituitary-thyroid axis.

There is a progressive shift in the serum TSH distribution curve towards higher TSH values with increasing age [2]. Many endocrine systems exhibitchanges with aging in the absence of overt disease thus age-related fall in circulating $\mathrm{T} 4$, reduction in TSH bioactivity or reduced responsiveness of the thyroid to TSH could result in increased TSH secretion. Another possibility is that it may be due to occult thyroid disease in older people or simply an age- related alteration in TSH set point [8,9]. It might be possible that variation in the level of serum TSH is not great enough (nonsignificant), as the endocrine functions those are essential to life, such as adrenal and thyroid functions, show a minimal overall change in basal levels with ageing $[9,16]$.

Rojas et al. found higher TSH level in postmenopausal women compared to premenopausal women [7]. They found average TSH values increased with age, although the changes between groups were not significant. American study by Pearce et al point towards increased TSH levels in post menopausal women [4].

Hollowell et alfound higher TSH levels in women in the older age group in the National Health and Nutrition Examination Survey - NHANES conducted in United States on large population [5].

An Italian study by Mariotti et al. found significantly higher TSH levels in younger women and claim that due to an age-related decrease in TSH secretion by the pituitary [10]. Hershmanet al found the mean TSH in older women was slightly but signi-ficantly lower than that in middle-aged women[11].

Limitations of the study was small sample size of geriatric population.

\section{Conclusion}

Subclinical hypothyroidism was observed in post menopausal women in our setting. A higher tendency of women having subclinical hypothyroidism compared to men was also observed. This suggests a necessity to assess functional status of thyroid gland periodically in the post menopausal period.

\section{Funding: Nil, Conflict of interest: None Permission of IRB: Yes}

Authors contribution: Dr G.S Chandrashekhar is the only author for the article, who planned the study, carried out the research and prepared the manuscript.

What this study adds to existing Knowledge: The present study contributes to the existing knowledge as it suggests a routine screening of thyroid function tests in postmenopausal women. 


\section{References}

1. Cooper DS. Thyroid disease in the oldest old: the exception to the rule. JAMA. 2004 Dec 1;292(21):26514. DOI:10.1001/jama.292.21.2651

2. Surks MI, Ortiz E, Daniels GH, et al. Subclinical thyroid disease: scientific review and guidelines for diagnosis and management. JAMA. 2004 Jan 14;291 (2): 228-38.DOI:10.1001/jama.291.2.228

3. Suzuki S, Nishio S, Takeda T, Komatsu M. Genderspecific regulation of response to thyroid hormone in aging. Thyroid Res. 2012 Jan 26;5(1):1. doi: 10.1186/ 1756-6614-5-1.

4. Pearce NE. Thyroid dysfunction in perimenopausal and postmenopausal women. Boston University Medical Center, Boston, Massachusetts, USA Menopause Int. 2007;13(1):8-13.

5. Hollowell JG, Staehling NW, Flanders WD, et al. Serum TSH, T(4), and thyroid antibodies in the United States population (1988 to 1994): National Health and Nutrition Examination Survey (NHANES III). J Clin Endocrinol Metab. 2002 Feb; 87 (2): 489-99.DOI:10. 1210 / jcem.87.2.8182

6. Usha Adiga. Thyroid Status, Renal Profile and Electrolytes in Postmenopausal Women. International Journal of Biochemistry Research and Review 2017;18 (1):1-5.

7. Rojas LV, Nieves K, Suarez E, et al. Thyroidstimulating hormone and follicle-stimulating hormone status in Hispanic women during the menopause transition.Ethn Dis.2008 Spring;18(2 Suppl 2):S2-230-4

8. Benhadi N, Fliers E, Visser TJ, et al. Pilot study on the assessment of the setpoint of the hypothalamuspituitary-thyroid axis in healthy volunteers. Eur $\mathrm{J}$ Endocrinol. 2010 Feb;162(2):323-9. doi: 10.1530/EJE09-0655. Epub 2009 Nov 19.
9. Chahal HS, Drake WM. The endocrine system and ageing. J Pathol. 2007 Jan;211(2):173-80.DOI:10.1002/ path. 2110

10. Mariotti S, Barbesino G, Caturegli P, et al. Complex alteration of thyroid function in healthy centenarians. J Clin Endocrinol Metab. 1993 Nov;77(5):1130-4.DOI: 10.1210/jcem.77.5.8077303

11. Hershman JM, Pekary AE, Berg L, et al. Serum thyrotropin and thyroid hormone levels in elderly and middle-aged euthyroid persons. J Am Geriatr Soc. 1993 Aug; 41(8):823-8.

12. Heuck CC, Kallner A, Kanagasabapathy AS, Riesen W. WHO Document (WHO/DIL /00.4) on "Diagnosis and monitoring of diseases of the thyroid", 2000.

13. Demers LM, Spencer CA. Laboratory medicine practice guidelines: laboratory support for the diagnosis and monitoring of thyroid disease. Clin Endocrinol (Oxf). 2003 Feb;58(2):138-40.

14. Garber JR, Cobin RH, Gharib H, et al. Clinical practice guidelines for hypothyroidism in adults: cosponsored by the American Association of Clinical Endocrinologists and the American Thyroid Association. EndocrPract. 2012 Nov-Dec; 18(6):9881028. DOI:10.4158/EP12280.GL

15. Brabant G, Prank K, Ranft U, et al. Physiological regulation of circadian and pulsatile thyrotropin secretion in normal man and woman. J Clin Endocrinol Metab. 1990 Feb;70(2):403-9.DOI:10. 1210 / jcem-70-2-403

16. Kuokkanen, S, Santoro, N. Endocrinology of the Perimenopausal Woman. Glob. libr. women's med. (ISSN: 1756-2228) 2011; doi: 10. 3843/ GLOWM. 10082.

\section{How to cite this article?}

G.S Chandrashekhar. Comparison of thyroid profile in premenopausal and postmenopausal women: A case control study. Int J Med Res Rev 2018; 6(07):367-371. doi:10.17511/ijmrr.2018.i07.05. 